\title{
Altura de lâmina e tempo de permanência de água na umidade de subs- tratos em subirrigação
}

\author{
Rhuanito S Ferrarezi ${ }^{1}$; Antonio C Ferreira Filho²; Roberto Testezlaf ${ }^{2}$ \\ ${ }^{1}$ University of Florida, Indian River Research and Education Center (UF-IFAS-IRREC), Fort Pierce-FL, United States; rferrarezi@ufl. \\ edu (corresponding author); ${ }^{2}$ Universidade Estadual de Campinas (UNICAMP), Campinas-SP, Brasil; antoniocff@gmail.com; bob@ \\ feagri.unicamp.br
}

\section{RESUMO}

A subirrigação reduz o descarte inadequado de água e nutrientes nos solos e lençóis freáticos por ser um sistema fechado, com recirculação de solução nutritiva. O sistema é utilizado comercialmente na indústria de plantas ornamentais. Porém, informações sobre a retenção de umidade em substratos utilizados em subirrigação ainda são escassas. Objetivou-se avaliar o efeito de diferentes alturas de lâmina e tempos de permanência de água na umidade volumétrica de substratos comerciais em subirrigação. Testaram-se cinco alturas de lâminas (1, 2, 4, 6 e $8 \mathrm{~cm})$, cinco tempos de permanência de água $(2,5 ; 5 ; 10 ; 15$ e $20 \mathrm{~min})$ e três substratos comerciais (fibra de coco, casca de pinus com vermiculita e turfa com casca de arroz carbonizada sem cinzas e vermiculita), arranjados como fatorial $5 \times 5 \times 3$, em delineamento inteiramente casualizado, com três repetições. Tubetes cônicos de $55 \mathrm{~cm}^{3}$ foram divididos em três frações iguais de 4,16 $\mathrm{cm}$ (inferior, intermediária e superior) para estudar a dinâmica da ascensão capilar da água. Os substratos apresentaram variação da umidade nas três frações dos tubetes $(p<0,0001)$. A umidade variou em função da altura da lâmina $\left(r^{2}>0,9\right)$, com pouca ou nenhuma influência do tempo de permanência de água. A fração intermediária apresentou melhor diferenciação entre os tratamentos, com variação de umidade de 0,29 a $0,61 \mathrm{~m}^{3} / \mathrm{m}^{3}$ na fibra de coco; 0,29 a $0,63 \mathrm{~m}^{3} / \mathrm{m}^{3}$ na casca de pinus e 0,24 a $0,56 \mathrm{~m}^{3} / \mathrm{m}^{3}$ na turfa. A altura de lâmina de $4 \mathrm{~cm}$ com qualquer tempo de permanência na fibra de coco e turfa e a altura de lâmina de $2 \mathrm{~cm}$ e tempo de 10 ou 20 min na casca de pinus resultaram em umidade na faixa recomendada para cultivo da maioria das espécies vegetais $\left(0,4 \mathrm{~m}^{3} / \mathrm{m}^{3}\right)$. O conhecimento da dinâmica da ascensão capilar na subirrigação permite a otimização da altura da lâmina e do tempo de irrigação para aplicar água eficientemente e suprir a evapotranspiração das culturas.

Palavras-chave: ascensão capilar, conteúdo volumétrico de água, manejo da irrigação.

\section{ABSTRACT \\ Water height and irrigation time effect on substrate water content in subirrigation}

Subirrigation is a closed system which recirculates nutrient solution and reduces water and nutrient improper disposal in soils and groundwater. The system is used commercially by the ornamental industry. However, technical information regarding substrate moisture retention in subirrigation still scarce. Our study evaluated the effect of different water heights and irrigation time on volumetric water content (VWC) of commercial substrates in subirrigation. We tested five water heights $(1,2,4,6$ and $8 \mathrm{~cm})$, five irrigation times $(2.5,5$, 10,15 and $20 \mathrm{~min}$ ), and three commercial substrates (coconut coir, pine bark with vermiculite, and peat moss with carbonized rice hulls without ashes and vermiculite), arranged in a complete randomized design (factorial $5 \times 5 \times 3$ ), with three replications. We used $55-\mathrm{cm}^{3}$ cone-shaped containers divided into three 4.16 -cm equal layers (top, middle, and bottom) to study the water capillary action dynamics. All substrates presented a large range of VWC across the container layers $(p<0.0001)$. The substrate moisture varied according to the water height $\left(r^{2}>0.9\right)$, with little or no influence of the irrigation time. The middle layer provided the best moisture indication among treatments, with moisture ranging from 0.29 to $0.61 \mathrm{~m}^{3} / \mathrm{m}^{3}$ in coconut coir, 0.29 to $0.63 \mathrm{~m}^{3} / \mathrm{m}^{3}$ in pine bark, and 0.24 to $0.56 \mathrm{~m}^{3} / \mathrm{m}^{3}$ in peat moss with carbonized rice hulls. The water height of $4 \mathrm{~cm}$ with any irrigation time for coconut coir and peat moss, and the water height of $2 \mathrm{~cm}$ and irrigation time of 10 or $20 \mathrm{~min}$ for pine bark resulted in the moisture range recommended for most crops $\left(0.4 \mathrm{~m}^{3} / \mathrm{m}^{3}\right)$. The understanding of capillary action dynamics in subirrigation allows the optimization of water heights and irrigation time to apply water efficiently to meet the crop evapotranspiration.

Keywords: capillary action, irrigation management, volumetric water content.

(Recebido para publicação em 27 de janeiro de 2016; aceito em 25 de setembro de 2016) (Received on January 27, 2016; accepted on September 25, 2016)

\begin{abstract}
A subirrigação é uma técnica de irrigação que aplica água e nutrientes diretamente na base dos recipientes de cultivo, promovendo o molhamento do substrato por meio da ascensão capilar (Ferrarezi et al., 2015c). A quantidade de água e fertilizantes absorvidos depende da umidade do substrato e da
\end{abstract}

demanda hídrica e nutricional das plantas (Ferrarezi et al., 2015c). A água ou solução nutritiva excedente é coletada e reutilizada em irrigações subsequentes, constituindo um sistema fechado ou recirculante, eliminando o escoamento superficial e lixiviação e a possibilidade de contaminação ambiental da água su- perficial e lençóis freáticos em função do descarte inadequado de nutrientes (Dumroese et al., 2006; Martinetti et al., 2008).

Sistemas com recirculação da solução nutritiva como a subirrigação utilizam 33\% da água aplicada por aspersão e $50 \%$ da aplicada por gotejamento nos 
cultivos em vasos (Roeber, 2010). A tecnologia possibilita a obtenção de altas eficiências de irrigação (teoricamente $100 \%$ ) e aplicação de menor quantidade de água quando manejada adequadamente (Ribeiro et al., 2014). Ferrarezi et al. $(2015 \mathrm{c})$ realizaram uma detalhada revisão de literatura sobre a subirrigação, indicando a evolução da tecnologia, equipamentos, princípios de operação, aplicações, manejo e perspectivas.

A subirrigação proporciona precocidade de produção de plantas (Ferrarezi et al., 2013), aumento da produção por área (Rouphael et al., 2006), melhoria na qualidade e uniformidade de produção (Biernbaum, 1990), redução ou eliminação da lixiviação de água e nutrientes (van Iersel \& Kang, 2002; Zheng et al., 2004), suprimento adequado e uniforme de nutrientes (Laviola et al., 2007) e maior eficiência no uso de água e fertilizantes devido à ausência de escoamento superficial e lixiviação (Schmal et al., 2011). Além disso, possibilita a imposição de estresse hídrico para promover a produção de plantas compactas e para aclimatação de plantas a estresses durante o transporte, comercialização e plantio (Ferrarezi et al., 2015a). Porém, como todo sistema de irrigação, a subirrigação apresenta limitações, como potencial para concentração de sais e aumento da condutividade elétrica nas camadas superiores do substrato (Ferrarezi \& Testeslaf, 2016), elevado custo de implantação e manutenção em comparação com outros sistemas (Elliott, 1992; Uva et al., 1998), maior risco de propagação de patógenos, especialmente aqueles disseminados pela água (van der Gaag et al., 2001) e alta variação na umidade do substrato ao longo do tempo (Ferrarezi et al., 2015a, 2015b). Por se tratar de um sistema com pouca utilização no Brasil (Ferrarezi \& Testeslaf, 2016), há escassez de informações sobre o manejo hídrico para diferentes substratos comerciais e recipientes de cultivo, dificultando a sua recomendação para culturas de interesse econômico.

Dentre as principais características operacionais que definem o manejo hídrico em mesas de subirrigação, estão o estabelecimento da altura da lâmina de água e seu tempo de permanência em contato com o substrato para se atingir a umidade ideal para crescimento das plantas (Lopes et al., 2007). Lâminas insuficientes podem levar à aplicação de água na base do recipiente de cultivo numa altura que não permita a ascensão capilar até a parte superior. Tempos de permanência reduzidos podem afetar a eficiência de aplicação de água e permanência prolongada pode provocar anoxia. Gent \& McAvoy (2011) relacionaram a umidade volumétrica do substrato com diferentes tempos de permanência de água ( 2 a 20 min) em vasos de 10 e $15 \mathrm{~cm}$ de diâmetro sem plantas. No entanto, a altura máxima utilizada foi de $5 \mathrm{~cm}$ e o substrato utilizado era à base de turfa e perlita $(83 \%: 17 \% \mathrm{v} / \mathrm{v})$, que apresenta uso reduzido no Brasil.

Em razão da diversidade de recipientes de cultivo e substratos utilizados na produção em ambiente protegido, informações técnicas relacionadas ao manejo da subirrigação são necessárias para viabilizar a sua aplicação em diferentes condições de produção. O uso de tubetes de $55 \mathrm{~cm}^{3}$ é padrão na produção de mudas de espécies florestais, porta-enxertos cítricos e eucalipto, e a subirrigação já foi testada com sucesso nessas culturas (Thebaldi, 2011; Ferrarezi et al., 2013; Ribeiro et al., 2014). Porém, ainda existe a necessidade de se conhecer a dinâmica de movimento vertical da água em substratos para otimizar a automação da subirrigação usando sensores capacitivos.

Sensores capacitivos possuem comprimentos variáveis, e o local de inserção dos sensores no substrato é determinante na leitura de umidade com acurácia. Além disso, a umidade estabelecida como referência para acionar a subirrigação exerce papel importante na manutenção adequada da umidade para crescimento vegetal. Ferrarezi et al. (2014) indicaram que a variação no conteúdo volumétrico de água (CVA) é maior quando os valores estabelecidos para acionamento da irrigação são baixos $\left(0,1\right.$ e $\left.0,2 \mathrm{~m}^{3} / \mathrm{m}^{3}\right)$. De acordo com esses autores, umidades de $0,5 \mathrm{~m}^{3} / \mathrm{m}^{3}$ para acionamento de mesas de subirrigação promoveram crescimento de sálvia (Salvia splendens) 233\% maior que a umidade de $0,1 \mathrm{~m}^{3} / \mathrm{m}^{3}$. A mesma tendência foi encontrada para massa seca, número de flores e ramos e área foliar (Ferrarezi et al., 2014). Ferrarezi et al. (2015a) indicaram que gradientes verticais de umidade ocorrem em recipientes subirrigados porque a água é absorvida lentamente pela fração inferior do substrato e se move verticalmente por ascensão capilar. As propriedades físicas dos substratos (densidade, estrutura das partículas, porosidade e capacidade de retenção de água) exercem influência no movimento de água (Caron et al., 2005; Ferrarezi et al., 2015c). A maioria dos substratos usados nas pesquisas com subirrigação (principalmente esfagno, turfa e lã de rocha) não são comuns no Brasil. Informações sobre a dinâmica da ascensão capilar da água em substratos como fibra de coco, casca de pinus e turfa com casca de arroz carbonizada ainda são escassas, principalmente em recipientes longos e de diâmetro reduzido como é o caso dos tubetes, sendo necessárias para orientar o uso da subirrigação nas condições brasileiras.

A hipótese deste trabalho é que o movimento vertical de água apresentará diferenças em substratos com composições variadas, havendo estratificação em razão de a subirrigação saturar a base dos recipientes de cultivo e a parte superior estar diretamente exposta ao ar. O conhecimento da dinâmica da umidade em diferentes substratos permitirá indicar a melhor camada para inserção de sensores para monitoramento da umidade em tempo real e estabelecer o manejo hídrico para atender às exigências de plantas cultivadas em tubetes dentro de ambientes protegidos.

O objetivo foi avaliar o efeito de diferentes alturas de lâmina e tempos de permanência de água na umidade volumétrica de substratos comerciais em subirrigação.

\section{MATERIAL E MÉTODOS}

O experimento foi realizado de janeiro a abril de 2012 na Faculdade de Engenharia Agrícola da UNICAMP, Campinas-SP (22\%49'12"S; $47^{\circ} 03^{\prime} 41^{\prime \prime} \mathrm{O} ; 640 \mathrm{~m}$ de altitude). $\mathrm{O}$ estudo foi conduzido em ambiente protegido, numa estufa modelo Poly-Venlo (Van der Hoeven, Holambra-SP), com 
dimensões de 18,2×6,4×3,0 m (largura $\times$ comprimento $\times$ altura), cobertura de polietileno de $150 \mu \mathrm{m}$ e fechamento das laterais e frente com tela antiafídea de $0,87 \times 0,30 \mathrm{~mm}$. Não havia controle das condições climáticas no interior da estufa. A umidade relativa e a temperatura do ar foram monitoradas a cada 15 min durante as coletas, utilizando-se um termo higrômetro (HT-4000; ICEL, Manaus, AM) (Tabela 1). O déficit de pressão de vapor (DPV, $\mathrm{kPa}$ ) foi calculado pelas equações 1 a 3 .

$\mathrm{DPV}=\mathrm{e}_{\text {sat }}-\mathrm{e}$

onde DPV = déficit de pressão de vapor $(\mathrm{kPa}) ; \mathrm{e}_{\mathrm{sat}}=$ pressão de saturação de vapor $(\mathrm{kPa})$ e e $=$ pressão atual de vapor $(\mathrm{kPa})$.

$$
\mathrm{e}_{\text {sat }}=0,6112 \times \exp ^{\mathrm{t}}
$$

onde $\mathrm{t}=(17,67 \mathrm{x}$ temperatura $) /($ temperatura $+243,5)$

$\mathrm{e}=\mathrm{e}_{\text {sat }} \times \frac{\text { Umidade relativa }}{100}$

onde $\mathrm{e}_{\text {sat }}=$ pressão de saturação de vapor $(\mathrm{kPa})$ e e $=$ pressão atual de vapor $(\mathrm{kPa})$.

$\mathrm{O}$ experimento foi conduzido em uma mesa de subirrigação tipo $e b b$ -and-flow de fibra de vidro (Redelease, São Paulo-SP) com dimensões de $1,0 \times 1,4 \times 0,1 \mathrm{~m}$, um conjunto moto bomba de 0,85 CV (Hydrobloc C800T; KSB Bombas e Válvulas, Manaus-AM), um reservatório de $150 \mathrm{~L}$ (FortLev, Camaçari-BA) e uma chave de partida de $1 \mathrm{CV}$ 220V monofásica (PDWM02; WEG, Jaraguá do Sul-SC) para acionamento do sistema (Figura 1a). O conjunto moto bomba foi conectado à mesa de subirrigação por tubulações e registros de PVC com diâmetro de $2,54 \mathrm{~cm}$ para permitir a aplicação das diferentes alturas de lâminas e tempos de permanência de água. Adicionalmente, a mesa possuía uma tubulação de drenagem com diâmetro de $2,54 \mathrm{~cm}$ que permitia o retorno da solução nutritiva ao reservatório para reutilização (Figura 1a).

Os tratamentos consistiram nas combinações de cinco alturas de lâmina $(1,2,4,6$ e $8 \mathrm{~cm})$, cinco tempos de permanência de água $(2,5 ; 5 ; 10 ; 15$ e $20 \mathrm{~min}$ ) e três substratos comerciais [fibra de coco (Golden Mix; Amafibra, Ananindeua, PA), casca de pinus com vermiculita na proporção $75 \%: 25 \%$, v/v (Tropstrato HT Hortaliças; Vida
Verde, Mogi Mirim-SP) e turfa de esfagno com casca de arroz carbonizada sem cinzas e vermiculita na proporção 62\%:18\%:20\%, v/v (Carolina Soil II; Carolina Soil do Brasil, Vera Cruz-RS)]. Utilizou-se fatorial $5 \times 5 \times 3$, no delineamento inteiramente casualizado, com três repetições, totalizando 375 amostras por substrato. Em cada repetição foram utilizados cinco tubetes rígidos de polietileno com $55 \mathrm{~cm}^{3}$ e dimensões de $12,48 \mathrm{~cm}$ de altura $\times 2,9 \mathrm{~cm}$ de diâ- metro interno e seis estrias (Aluminox, Jundiaí-SP).

Os substratos foram caracterizados fisicamente antes do início dos experimentos. A densidade aparente $\left(\rho_{\text {ap }}\right)$ [Eq. 4], densidade real ( $\left.\rho_{\text {real }}\right)$ [Eq. 5] e porosidade total $(\alpha)$ [Eq. 6] dos substratos foram determinadas de acordo com metodologia proposta pela Embrapa (2005) (Tabela 2). A capacidade de retenção de água (CRA) [Eq. 7] foi determinada de acordo com Pire \& Pereira (2003)

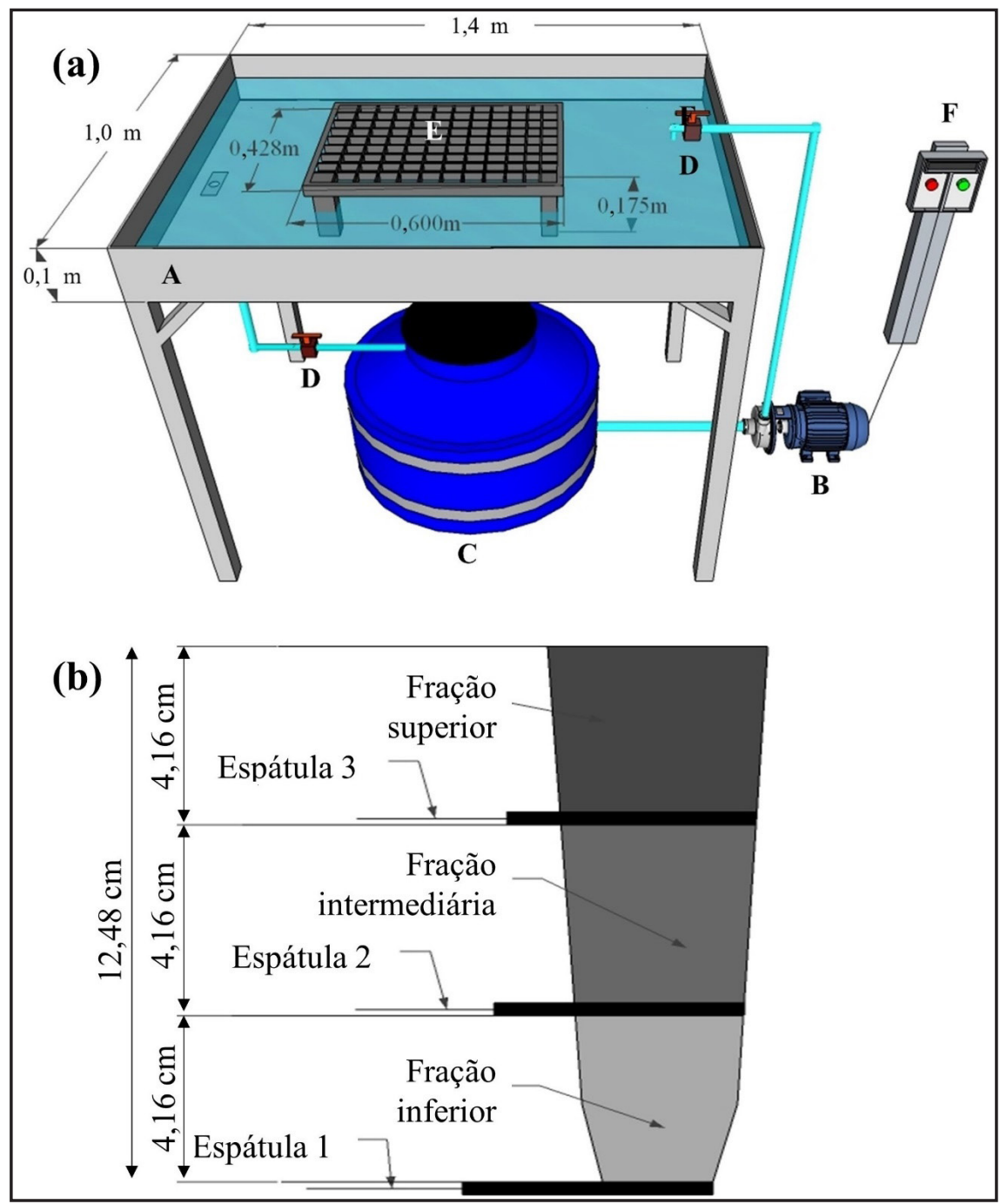

Figura 1. Módulo experimental (a), formado por A) Mesa de subirrigação tipo ebb-and-flow, B) Conjunto moto bomba de $0,85 \mathrm{CV}, \mathrm{C}$ ) reservatório de $150 \mathrm{~L}, \mathrm{D})$ registros rápidos, E) bandeja plástica para suporte dos tubetes, e F) chave de partida para acionamento do sistema. Frações dos tubetes de $55 \mathrm{~cm}^{3}(12,48 \mathrm{~cm}$ de altura $\times 2,9 \mathrm{~cm}$ de diâmetro) (b), indicando onde os mesmos foram cortados para individualização das amostras. A fração da base a 4,16 $\mathrm{cm}$ foi denominada inferior, a fração de 4,17 a $8,32 \mathrm{~cm}$ foi denominada intermediária e a fração de 8,33 a 12,48 cm foi denominada superior \{experimental setup (a) consisting of A) ebb-and-flow subirrigation bench, B) 0.83-HP irrigation pump, C) 150-L water tank, D) shut off valve, E) plastic tray to support the cone-shaped containers, and F) on/off switch. $55-\mathrm{cm}^{3}$ cone-shaped container $(12.48 \mathrm{~cm}$ height $\times 2.9 \mathrm{~cm}$ diameter) layers (b), indicating the three individual samples. The fraction from the base to $4.16 \mathrm{~cm}$ was named bottom layer, the fraction from 4.17 to $8.32 \mathrm{~cm}$ was named intermediate layer, and the fraction from 8.33 to $12.48 \mathrm{~cm}$ was named top layer . Campinas, UNICAMP, 2012. 
(Tabela 2).

$\rho_{\mathrm{ap}}=\frac{\text { peso amostra seca }}{\text { volume recipiente }}$

onde $\rho_{\text {ap }}=$ densidade aparente $\left(\mathrm{g} / \mathrm{cm}^{3}\right)$,

$\rho_{\text {real }}=\frac{\text { peso amostra seca }}{\text { volume amostra }}$

onde $\rho_{\text {real }}=$ densidade real $(\mathrm{g} / \mathrm{L})$

$\alpha=\frac{1-\rho_{\text {ap }}}{\rho_{\text {real }}} \times \times \cdot 100$

onde $\alpha=$ porosidade total $(\%)$

$\mathrm{CRA}=\frac{\mathrm{p} \text { úmida }-\mathrm{p} \text { seca }}{\text { volume recipiente }}$

onde $\mathrm{CRA}=$ capacidade de retenção de água $\left(\mathrm{m}^{3} / \mathrm{m}^{3}\right) ; \mathrm{p}$ úmida $=$ peso da amostra úmida e p seca $=$ peso da amostra seca.

A umidade volumétrica inicial dos três substratos foi uniformizada em $0,2 \mathrm{~m}^{3} / \mathrm{m}^{3}$, usando como referência a umidade inicial do substrato de casca de pinus com vermiculita. Os fabricantes dos outros substratos indicam a necessidade de reconstituição da umidade antes do uso. Para atingir esse valor, na fibra de coco adicionou-se $1 \mathrm{~L}$ de água para cada $\mathrm{kg}$ de substrato, enquanto que na casca de arroz carbonizada sem cinzas e vermiculita adicionaram-se $5 \mathrm{~L}$ de água em $45 \mathrm{~L}$ de substrato.

Os tubetes foram seccionados em três frações iguais de 4,16 cm (Figura 1b), denominadas, da base ao ápice, de inferior, intermediária e superior (Figura 1b). Esse fracionamento foi necessário para se estudar a dinâmica da ascensão capilar da água nos tubetes, e seus valores foram baseados em resultados prévios usando bandejas de subirrigação para a produção de porta-enxertos cítricos (Barreto et al., 2012). $\mathrm{Na}$ realização dos experimentos, as frações eram unidas com fita adesiva para que a altura do tubete fosse mantida e que não houvesse perda de água nas paredes do recipiente. $\mathrm{Na}$ base de cada tubete colocou-se uma tela plástica circular com $8 \mathrm{~cm}$ de diâmetro e orifícios de $2 \mathrm{~mm}$ com o auxílio de um elástico, para evitar perda de substrato durante $\mathrm{o}$ processo de umedecimento. Os tubetes foram preenchidos com os substratos usando o método indicado por Pire \& Pereira (2003), que requer quatro impactos numa superfície plana para o tubete ficar totalmente cheio e compactado (Barreto et al., 2012). Utilizou-se como compactação de referência a mesma usada no cultivo comercial de plantas.

Após a fixação da primeira altura da lâmina de água na mesa de subirrigação, 25 tubetes foram colocados numa bandeja plástica plana de 42,8×60,5×2,9 cm com 104 células (Aluminox, Jundiaí-SP) para as coletas das amostras ao longo do tempo (cinco tubetes para cada tempo). As bandejas foram apoiadas em pés móveis com 17,5 cm de altura, que foram cortados na altura de $13,5 \mathrm{~cm}$ (os tubetes ficaram afastados em $1 \mathrm{~cm}$ da base da mesa de subirrigação). Os tubetes foram retirados em grupos de cinco nos respectivos tempos de permanência de água estabelecidos pelos tratamentos. Com o auxílio de espátulas (Figura 1b), as amostras das diferentes frações do tubete foram retiradas e acondicionadas em latas de alumínio de $100 \mathrm{~cm}^{3}$ com tampa hermética para evitar perda de umidade. Esse processo foi repetido para cada altura de lâmina.

A umidade do substrato foi determinada por gravimetria, secando-se as amostras em estufa de circulação forçada de ar a $65^{\circ} \mathrm{C}$ por 48 horas e pesando-as em balança de precisão com quatro casas decimais. A partir do peso úmido e seco calculou-se a umidade gravimétrica (UG) [Eq. 8] e a umidade volumétrica $(\Theta)$ [Eq. 9].

$\mathrm{UG}=\frac{\text { água na amostra }}{\text { peso amostra seca }}$

onde $\mathrm{UG}=$ umidade gravimétrica $(\mathrm{g} / \mathrm{g})$

$\theta=U G \times \rho_{\mathrm{ap}}$

onde $\Theta=$ conteúdo volumétrico de água do substrato $\left(\mathrm{m}^{3} / \mathrm{m}^{3}\right)$.

Os resultados foram submetidos à análise de variância e de regressão usando o software estatístico SAS (v. 9.2; SAS Institute, Cary, NC, Estados Unidos). Para determinar os efeitos das alturas da lâmina (AL) e dos tempos de permanência de água (TPA) no conteúdo volumétrico de água (CVA) dos substratos, foi usado um modelo de regressão que incluía os efeitos principais, sua interação e componentes quadráticos: $\mathrm{CVA}=\mathrm{a}_{0}+\left(\mathrm{a}_{1} \times \mathrm{AL}\right)+\left(\mathrm{a}_{2} \times \mathrm{AL}^{2}\right)+$ $\left(\mathrm{a}_{3} \times \mathrm{TPA}\right)+\left(\mathrm{a}_{4} \times \mathrm{TPA}^{2}\right)+\left(\mathrm{a}_{5} \times \mathrm{AL} \times\right.$ TPA), onde $a_{0}, \ldots, a_{5}=$ coeficientes de regressão. Os resultados foram considerados significativos a $5 \%(p<0,05)$. Os componentes não significativos da equação de regressão foram eliminados usando a ferramenta stepwise. Os coeficientes de determinação $\left(r^{2}\right)$ parciais, que indicam quanto cada fator testado contribuiu com os resultados para cada variável, foram obtidos com a instrução scorr1(seqtests).

\section{RESULTADOS E DISCUSSÃO}

Houve efeito dos tratamentos testados no CVA de todas as frações do tubete (Tabela 3), com exceção da interação substrato $\times$ tempo de permanência de água e substrato $\times$ altura de lâmina $\times$ tempo de permanência de água na fração inferior. Esse resultado provavelmente foi consequência do processo de saturação que ocorreu na fração inferior do tubete, com respostas diferentes causadas por substratos com características físicas distintas.

À medida que se aumentou a altura da lâmina de água, verificou-se aumento da umidade nas três frações do tubete na

Tabela 1. Variáveis ambientais [umidade relativa, temperatura do ar e déficit de pressão de vapor (DPV)] durante a coleta de dados dos substratos fibra de coco, casca de pinus com vermiculita e turfa com casca de arroz carbonizada sem cinzas e vermiculita \{environmental conditions [relative humidity, air temperature, and vapor pressure deficit (DPV)] during data collection of coconut coir, pine bark with vermiculite and peat with carbonized rice husk without ashes and vermiculite potting mixes\}. Campinas, UNICAMP, 2012.

\begin{tabular}{|c|c|c|c|c|c|}
\hline \multirow{2}{*}{ Substratos } & \multirow{2}{*}{$\begin{array}{c}\text { Umidade } \\
\text { relativa }(\%)\end{array}$} & \multicolumn{3}{|c|}{ Temperatura $\left({ }^{\circ} \mathrm{C}\right)$} & \multirow{2}{*}{$\begin{array}{l}\text { DPV } \\
\text { (kPa) }\end{array}$} \\
\hline & & Mínima & Média & Máxima & \\
\hline Fibra de coco & 33 & 25,4 & 27,9 & 36,2 & 2,52 \\
\hline $\begin{array}{l}\text { Casca de pinus com } \\
\text { vermiculita }\end{array}$ & 33 & 33,7 & 34,5 & 40,7 & 3,67 \\
\hline $\begin{array}{l}\text { Turfa com casca de } \\
\text { arroz carbonizada sem } \\
\text { cinzas e vermiculita }\end{array}$ & 46 & 29,8 & 31,6 & 32,7 & 2,51 \\
\hline
\end{tabular}


fibra de coco (Figura 2), casca de pinus com vermiculita (Figura 3) e turfa com casca de arroz carbonizada sem cinzas e vermiculita (Figura 4). A umidade variou nas três frações do tubete em resposta à altura da lâmina ( $\mathrm{AL}$ e $\left.\mathrm{AL}^{2}\right)$, com pouca influência no tempo de permanência de água (TPA e $\mathrm{TPA}^{2}$ ) e da interação AL $\times$ TPA (Tabela 4). Na fração intermediária do substrato de fibra de coco, a AL foi responsável por $90 \%$ dos resultados $\left(r^{2}=0,9014\right)$. O mes-

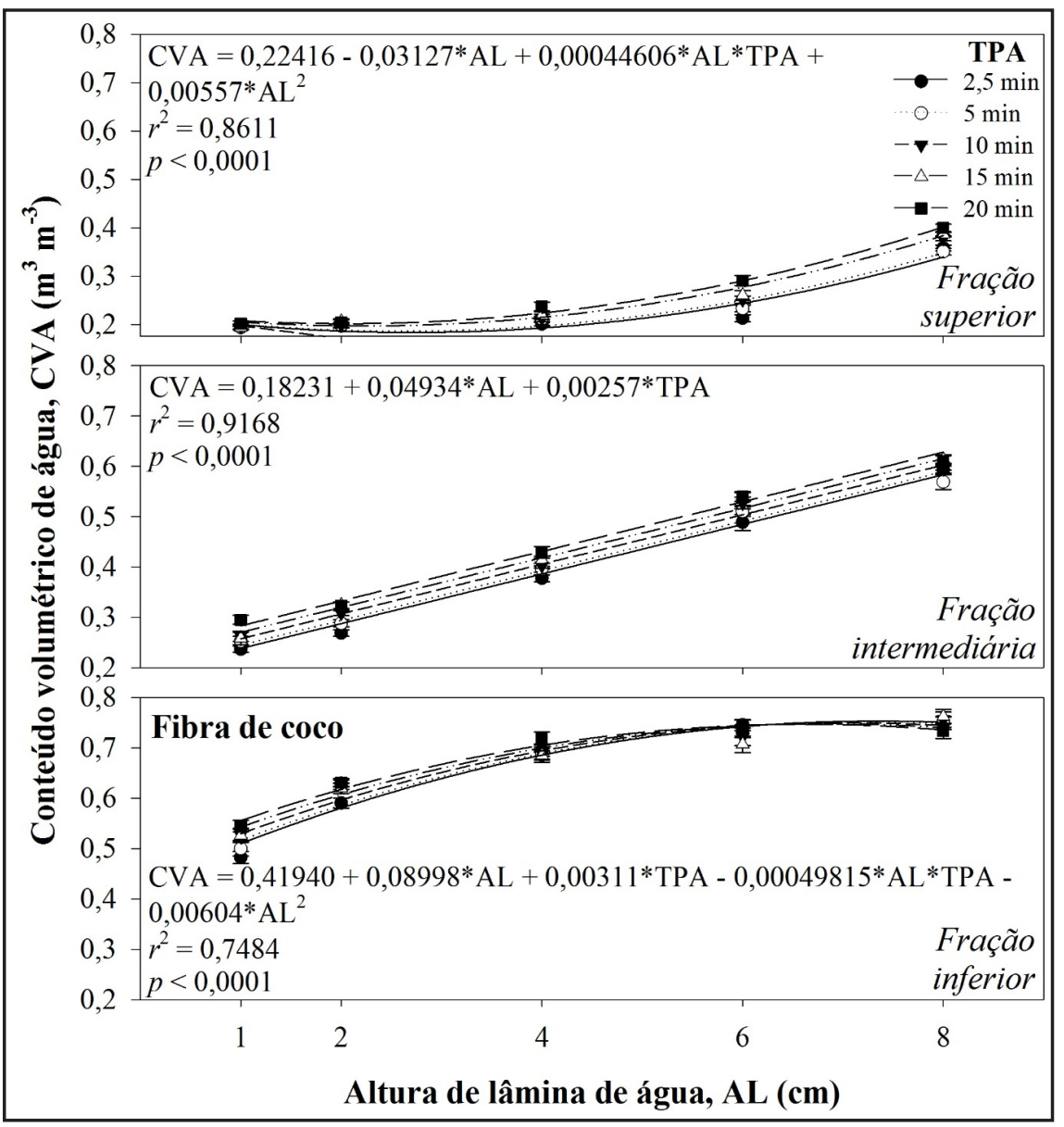

Figura 2. Conteúdo volumétrico de água (CVA) do substrato fibra de coco em função da altura de lâmina (AL) e do tempo de permanência de água (TPA) para cada fração dos tubetes (superior, intermediária e inferior). Média \pm erro padrão de cinco tubetes e três repetições \{volumetric water content (CVA) of coconut coir potting mix based on water height (AL) and irrigation time (TPA) for each container layer (top, middle, and bottom). Mean \pm standard error of five containers and three replications $\}$. Campinas, UNICAMP, 2012.

Tabela 2. Densidade aparente $\left(\rho_{\text {ap }}\right)$, densidade real $\left(\rho_{\text {real }}\right)$, porosidade total $(\alpha)$ e capacidade de retenção de água (CRA) dos substratos fibra de coco, casca de pinus com vermiculita e turfa com casca de arroz carbonizada sem cinzas e vermiculita \{bulk density $\left(\rho_{\text {ap }}\right)$, real density $\left(\rho_{\text {real }}\right)$, total porosity $(\alpha)$ and water holding capacity (CRA) of coconut coir, pine bark with vermiculite and peat with carbonized rice husk without ashes and vermiculite potting mixes \}. Campinas, UNICAMP, 2012.

\begin{tabular}{|c|c|c|c|c|}
\hline \multirow{2}{*}{ Substratos } & \multicolumn{2}{|c|}{ Densidade $\left(\mathrm{g} / \mathrm{cm}^{3}\right)$} & \multirow{2}{*}{$\begin{array}{l}\text { Porosidade } \\
\text { total, } \alpha(\%)\end{array}$} & \multirow{2}{*}{$\begin{array}{c}\text { CRA } \\
\left(\mathrm{m}^{3} / \mathrm{m}^{3}\right)\end{array}$} \\
\hline & Aparente, $\rho_{\text {ap }}$ & Real, $\rho_{\text {real }}$ & & \\
\hline Fibra de coco & 0,068 & 0,404 & 83,2 & 0,66 \\
\hline $\begin{array}{l}\text { Casca de pinus com } \\
\text { vermiculita }\end{array}$ & 0,41 & 1,47 & 72,10 & 0,54 \\
\hline $\begin{array}{l}\text { Turfa com casca de } \\
\text { arroz carbonizada sem } \\
\text { cinzas e vermiculita }\end{array}$ & 0,105 & 0,818 & 87,2 & 0,40 \\
\hline
\end{tabular}

mo ocorreu com a casca de pinus com vermiculita, na qual AL $\left(r^{2}=0,8392\right)$ e $\mathrm{AL}^{2}\left(r^{2}=0,0102\right)$ foram responsáveis por $85 \%$ dos resultados, e com a turfa com casca de arroz carbonizada sem cinzas e vermiculita, em que $\operatorname{AL}\left(r^{2}=0,9408\right)$ e $\mathrm{AL}^{2}\left(r^{2}=0,0037\right)$ foram responsáveis por $94 \%$ dos resultados.

O CVA da fibra de coco variou de 0,48 a $0,76 \mathrm{~m}^{3} / \mathrm{m}^{3}$ na fração inferior $\left(r^{2}=0,7484\right)$, de 0,29 a $0,61 \mathrm{~m}^{3} / \mathrm{m}^{3}$ na fração intermediária $\left(r^{2}=0,9168\right)$ e de 0,19 a $0,40 \mathrm{~m}^{3} / \mathrm{m}^{3}$ na fração superior $\left(r^{2}=\right.$ 0,8611) (Figura 2). Valores semelhantes foram encontrados por Ferrarezi et al. (2012), que observaram variação de 0,24 a $0,72 \mathrm{~m}^{3} / \mathrm{m}^{3}$ na subirrigação por pavio usando o mesmo substrato à base de fibra de coco. O CVA da casca de pinus com vermiculita variou de 0,56 a 0,71 $\mathrm{m}^{3} / \mathrm{m}^{3}$ na fração inferior $\left(r^{2}=0,5983\right)$, de 0,29 a $0,63 \mathrm{~m}^{3} / \mathrm{m}^{3}$ na fração intermediária $\left(r^{2}=0,9014\right)$ e de 0,23 a $0,44 \mathrm{~m}^{3} / \mathrm{m}^{3}$ na fração superior $\left(r^{2}=0,8335\right)$ (Figura 3 ). Esses valores foram menores que os encontrados por Ferrarezi et al. (2012), variando de 0,42 a $0,94 \mathrm{~m}^{3} / \mathrm{m}^{3}$, para a subirrigação por pavio usando o mesmo substrato à base de casca de pinus com vermiculita. O CVA da turfa com casca de arroz carbonizada sem cinzas e vermiculita variou de 0,44 a $0,74 \mathrm{~m}^{3} / \mathrm{m}^{3}$ na fração inferior $\left(r^{2}=0,8222\right)$, de $0,24 \mathrm{a}$ $0,56 \mathrm{~m}^{3} / \mathrm{m}^{3}$ na fração intermediária $\left(r^{2}=\right.$ 0,9568 ) e de 0,2 a $0,35 \mathrm{~m}^{3} / \mathrm{m}^{3}$ na fração superior $\left(r^{2}=0,8644\right)$ (Figura 4$)$.

As frações inferiores dos tubetes $(0$ a 4,16 cm) mantiveram-se em contato direto com a água durante a subirrigação, justificando o teor mais elevado de umidade em comparação com as outras frações. Na fibra de coco e na casca de pinus com vermiculita, o aumento das alturas de lâmina para valores maiores que $4 \mathrm{~cm}$ (Figuras 1 e 2) promoveu umidade acima da capacidade de retenção de água $\left(0,66\right.$ e $0,54 \mathrm{~m}^{3} / \mathrm{m}^{3}$, respectivamente) (Tabela 2), promovendo saturação dos substratos. O mesmo ocorreu na turfa com casca de arroz carbonizada sem cinzas e vermiculita, que apresentou umidade acima da capacidade de retenção de água $\left(0,4 \mathrm{~m}^{3} / \mathrm{m}^{3}\right)$ (Tabela 2) em todas as alturas de lâmina (Figura 4).

A camada superior dos tubetes $(8,33$ a $12,48 \mathrm{~cm}$ ) com fibra de coco e casca de pinus com vermiculita não atingiu 


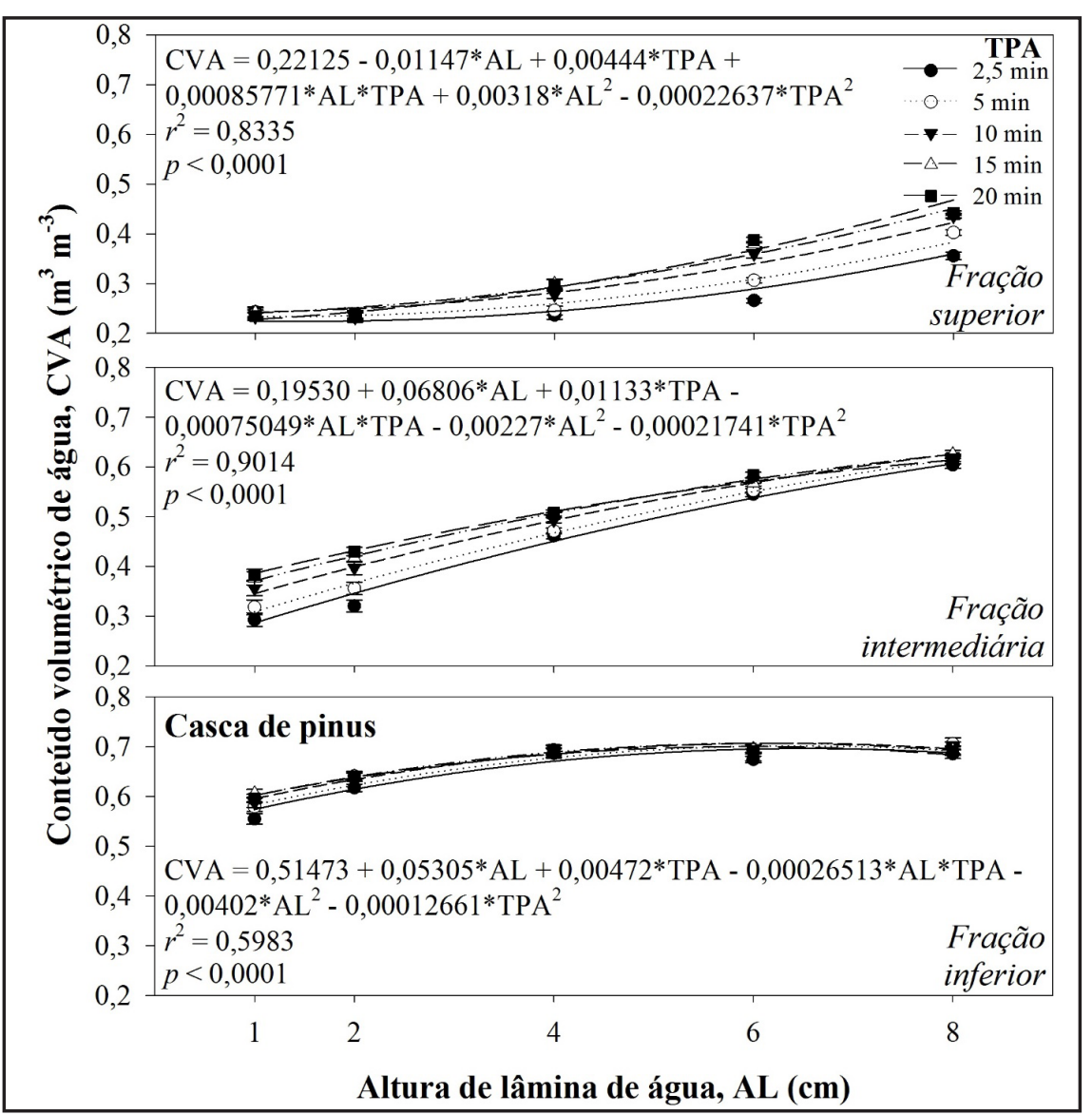

Figura 3. Conteúdo volumétrico de água (CVA) do substrato casca de pinus com vermiculita em função da altura de lâmina (AL) e do tempo de permanência de água (TPA) para cada fração dos tubetes (superior, intermediária e inferior). Média \pm erro padrão de cinco tubetes e três repetições \{volumetric water content (CVA) of pine bark with vermiculite potting mix based on water height (AL) and irrigation time (TPA) for each container layer (top, middle, and bottom). Mean \pm standard error of five containers and three replications $\}$. Campinas, UNICAMP, 2012.

Tabela 3. Análise de variância (ANOVA) do conteúdo volumétrico de água em função do substrato, altura da lâmina e tempo de permanência de água para cada fração do tubete (inferior, intermediária e superior). Foram usados cinco tubetes e três repetições para as análises \{analysis of variance (ANOVA) of the substrate volumetric water content in function of potting mix, water height and irrigation time for each container layer (top, middle, and bottom). We used five containers and three replications for the analysis\}. Campinas, UNICAMP, 2012.

\begin{tabular}{lcccc}
\hline \multirow{2}{*}{ Fonte de variação } & \multirow{2}{*}{$\begin{array}{c}\text { Graus de } \\
\text { liberdade }\end{array}$} & \multicolumn{3}{c}{ Frações do tubete } \\
\cline { 3 - 5 } & & \multicolumn{2}{c}{ Valor de probabilidade, $P r>\boldsymbol{F}$} \\
\cline { 3 - 5 } & 2 & $<0,0001$ & $<0,0001$ & $<0,0001$ \\
\hline Substrato (S) & 4 & $<0,0001$ & $<0,0001$ & $<0,0001$ \\
Altura da lâmina (AL) & 8 & $<0,0001$ & $<0,0001$ & $<0,0001$ \\
$\mathrm{~S} \times \mathrm{AL}$ & & $<0,0001$ & $<0,0001$ & $<0,0001$ \\
Tempo de permanência & 4 & 0,7446 & 0,0017 & $<0,0001$ \\
de água (TPA) & 8 & 0,0002 & $<0,0001$ & $<0,0001$ \\
$\mathrm{~S} \times \mathrm{TPA}$ & 16 & 0,6399 & 0,0220 & $<0,0001$ \\
$\mathrm{AL} \times \mathrm{TPA}$ & 32 & 0,8207 & 0,9367 & 0,8994 \\
$\mathrm{~S} \times \mathrm{AL} \times \mathrm{TPA}$ & & 6,22 & 7,53 & 9,25 \\
\hline$r^{2}$ & & 0,6394 & 0,4279 & 0,2638 \\
$\mathrm{CV}(\%)$ & & & & \\
Média $\left(\mathrm{m}^{3} / \mathrm{m}^{-3}\right)$ &
\end{tabular}

a umidade de saturação $(0,66$ e 0,54 $\mathrm{m}^{3} / \mathrm{m}^{3}$, respectivamente), mesmo com a máxima altura de lâmina $(8 \mathrm{~cm})$ e maior tempo de permanência de água (20 min) (Figuras 2 e 3). Esse fato pode ter sido consequência da sua exposição à atmosfera, sob condições de baixa umidade relativa do ar e elevadas temperaturas, o que pode ser comprovado pelos dados da Tabela 1. Além disso, de acordo com Zanetti et al. (2003), a capacidade, altura, formato e material de fabricação do recipiente de cultivo também exercem influência na relação entre o ar e a água dos substratos. Quanto maior a altura do recipiente utilizado, maior será o diferencial de potencial necessário para a água atingir as camadas superiores e menor a capacidade de água disponível, proporcionando menor uniformidade na umidade do substrato (Milner, 2001; Bailey et al., 2014). A altura do recipiente é fundamental na determinação na relação entre o espaço preenchido com água e ar no substrato, o que influenciará a retenção da água após a irrigação. Com o aumento da altura do recipiente, ocorre aumento nos espaços porosos preenchidos com ar devido ao aumento da drenagem causada pela ação da força gravitacional, provocando diminuição na capacidade de retenção de água (Bailey et al., 2014).

A camada superior nos tubetes com casca de arroz carbonizada sem cinzas e vermiculita não atingiu a umidade de $0,4 \mathrm{~m}^{3} / \mathrm{m}^{3}$ quando se usou altura da lâmina máxima $(8 \mathrm{~cm})$ e maior tempo de permanência de água (20 min) (Figura 4), em razão da alta porosidade desse substrato $(87,2 \%$, Tabela 2$)$. Com isso, houve limitação da ascensão capilar da água, o que pode causar redução na produção se essa fração for utilizada para controle da irrigação. Esses resultados foram contrários aos de Gent \& McAvoy (2011), que encontraram aumento exponencial da umidade volumétrica com o aumento do tempo de permanência da água na mesa de subirrigação, variando de 0,22 a $0,58 \mathrm{~m}^{3} / \mathrm{m}^{3}$ para tempos de 2 a $20 \mathrm{~min}$. Porém, aqueles autores realizaram a medição da umidade em todo o recipiente de cultivo, não havendo estratificação como no presente estudo. Além disso, o substrato de turfa e perlita utilizado (TG1; Lafaille and 


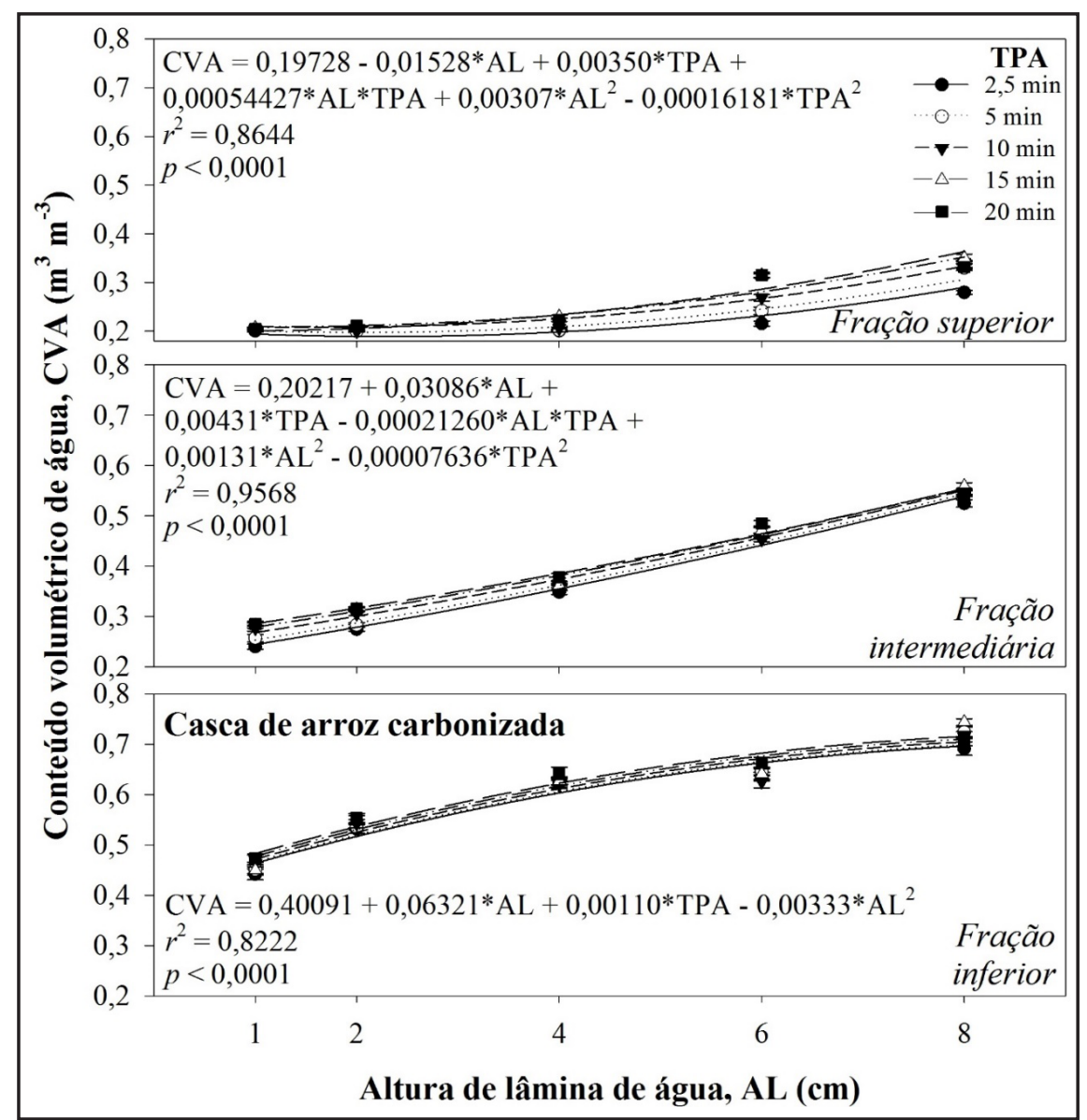

Figura 4. Conteúdo volumétrico de água (CVA) do substrato turfa com casca de arroz carbonizada sem cinzas e vermiculita em função da altura de lâmina (AL) e do tempo de permanência de água (TPA) para cada fração dos tubetes (superior, intermediária e inferior). Média \pm erro padrão de cinco tubetes e três repetições \{volumetric water content (CVA) of peat moss with carbonized rice hulls without ashes and with vermiculite potting mix based on water height (AL) and irrigation time (TPA) for each container layer (top, middle, and bottom). Mean \pm standard error of five containers and three replications $\}$. Campinas, UNICAMP, 2012.

Beaver Peat Co., Quebec, Canada) apresentava maior percentual de turfa na sua composição ( $83 \%)$ em comparação com o do nosso estudo (62\%), o que provavelmente resultou em maior ascensão capilar. De acordo com Barreto et al. (2012), substratos com textura fina apresentaram maior elevação de água por ascensão capilar e o substrato fino de fibra de coco apresentou os melhores resultados para aplicação na irrigação por capilaridade, recomendando-se a altura da lâmina de água de $5 \mathrm{~cm}$ por 15 min. A utilização de substratos com textura grossa de componentes granulares e fibrosos apresenta limitações para elevação de água por ascensão capilar devido à baixa capacidade de elevar a umidade em níveis de tensão de água disponível.
A fração intermediária dos tubetes $(4,17$ a $8,32 \mathrm{~cm})$ não atingiu umidade volumétrica próxima da saturação como a fração inferior. Nessa camada, a variação da umidade foi de 0,29 a $0,61 \mathrm{~m}^{3} / \mathrm{m}^{3}$ na fibra de coco, 0,29 a $0,63 \mathrm{~m}^{3} / \mathrm{m}^{3}$ na casca de pinus com vermiculita e 0,24 a $0,56 \mathrm{~m}^{3} / \mathrm{m}^{3}$ na turfa com casca de arroz carbonizada sem cinzas e vermiculita. A fibra de coco apresentou umidade de $0,4 \mathrm{~m}^{3} / \mathrm{m}^{3}$, valor considerado adequado para o desenvolvimento de plantas (Kim et al., 2011) na altura de lâmina de $4 \mathrm{~cm}$ (Figura 2). A casca de pinus com vermiculita apresentou umidade inferior a $0,4 \mathrm{~m}^{3} / \mathrm{m}^{3}$ para as alturas de lâminas de 1 e $2 \mathrm{~cm}$ (Figura 3). A turfa com casca de arroz carbonizada sem cinzas e vermiculita apresentou umidade superior a $0,4 \mathrm{~m}^{3} / \mathrm{m}^{3}$ a partir da altura de lâmina de $6 \mathrm{~cm}$ (Figura 4). No entanto, a ascensão capilar nesse substrato foi inferior à dos demais, provavelmente em razão da sua composição mais grosseira. A casca de arroz carbonizada reduz a ascensão capilar da mistura por causa da maior macroporosidade, resultando em menor capacidade de retenção de água. A maior altura dos tubetes em relação à sua largura também pode ter influenciado negativamente nesse resultado (Milner, 2001; Zanetti et al., 2003; Bailey et al., 2014). Como os substratos sofreram menor influência da perda de água por evaporação na superfície, a fração intermediária pode ser utilizada para o controle automatizado da subirrigação a partir do CVA. Isso possibilitará o emprego de menores alturas de lâmina e/ou tempo de permanência de água, reduzindo o tamanho da estrutura física e bombeamento necessários para aplicar água nas mesas de subirrigação.

Os resultados de umidade volumétrica foram semelhantes para a fibra de coco e casca de pinus com vermiculita nas três frações avaliadas (Figuras 3 e 4). Exceção ocorreu na fração inferior da fibra de coco, que apresentou maior umidade em comparação com a casca de pinus com vermiculita. Esses dados são diferentes dos encontrados por Barreto et al. (2012), nos quais o substrato de coco fino apresentou água disponível em todo o perfil do recipiente, enquanto o de pinus apresentou as camadas superiores do recipiente com água retida em tensões abaixo do ponto de murcha permanente. A altura dos tubetes também foi responsável por esses resultados, conforme indicado por Milner (2001), Zanetti et al. (2003) e Bailey et al. (2014) para o substrato à base de fibra de coco.

A umidade volumétrica dos substratos aumentou com a aplicação de alturas crescentes de lâmina. No entanto, o tempo de permanência de água apresentou pouca ou nenhuma influência nos resultados. A fração intermediária do tubete foi a mais adequada como indicadora da umidade do substrato, em razão do menor efeito da evaporação que ocorre na camada superior e da saturação da camada inferior. Obtiveram-se umidades do substrato maiores ou iguais a $0,4 \mathrm{~m}^{3} / \mathrm{m}^{3}$, com a utilização da altura 
Tabela 4. Coeficientes de determinação $\left(r^{2}\right)$ parciais e totais dos substratos fibra de coco, casca de pinus com vermiculita e turfa com casca de arroz carbonizada sem cinzas e vermiculita em função da altura de lâmina (AL) e do tempo de permanência de água (TPA) para cada fração do tubete (inferior, intermediária e superior) \{partial and total $\mathrm{r}^{2}$ coefficients of coconut coir, pine bark with vermiculite and peat with carbonized rice husk without ashes and vermiculite potting mixes based on water height (AL) and irrigation time (TPA) for each container layer (top, middle, and bottom)\}. Campinas, UNICAMP, 2012.

\begin{tabular}{|c|c|c|c|}
\hline Fator & Fração inferior & Fração intermediária & Fração superior \\
\hline & \multicolumn{3}{|c|}{ Fibra de coco, $r^{2}$ parcial } \\
\hline AL & 0,6448 & 0,9014 & 0,0400 \\
\hline $\mathrm{AL}^{2}$ & 0,0924 & --- & 0,7832 \\
\hline TPA & 0,0043 & 0,0154 & --- \\
\hline $\mathrm{TPA}^{2}$ & --- & --- & --- \\
\hline $\mathrm{AL} \times \mathrm{TPA}$ & 0,0069 & --- & 0,0379 \\
\hline \multirow[t]{2}{*}{$r^{2}$ total } & 0,7484 & 0,9168 & 0,8611 \\
\hline & \multicolumn{3}{|c|}{ Casca de pinus com vermiculita, $r^{2}$ parcial } \\
\hline $\mathrm{AL}$ & 0,4470 & 0,8392 & 0,0048 \\
\hline $\mathrm{AL}^{2}$ & 0,1317 & 0,0102 & 0,7363 \\
\hline TPA & 0,0082 & 0,0363 & 0,0051 \\
\hline $\mathrm{TPA}^{2}$ & 0,0051 & 0,0036 & 0,0033 \\
\hline $\mathrm{AL} \times \mathrm{TPA}$ & 0,0063 & 0,0121 & 0,0840 \\
\hline \multirow[t]{2}{*}{$r^{2}$ total } & 0,5983 & 0,9014 & 0,8335 \\
\hline & \multicolumn{3}{|c|}{$\begin{array}{c}\text { Turfa com casca de arroz carbonizada } \\
\text { sem cinzas e vermiculita, } r^{2} \text { parcial }\end{array}$} \\
\hline $\mathrm{AL}$ & 0,7876 & 0,9408 & 0,0214 \\
\hline $\mathrm{AL}^{2}$ & 0,0292 & 0,0037 & 0,7570 \\
\hline TPA & 0,0054 & 0,0106 & 0,0066 \\
\hline $\mathrm{TPA}^{2}$ & --- & 0,0005 & 0,0017 \\
\hline $\mathrm{AL} \times \mathrm{TPA}$ & --- & 0,0012 & 0,0777 \\
\hline$r^{2}$ total & 0,8222 & 0,9568 & 0,8644 \\
\hline
\end{tabular}

de lâmina de $4 \mathrm{~cm}$ com qualquer tempo de permanência para a fibra de coco e turfa com casca de arroz carbonizada sem cinzas e vermiculita, e altura de lâmina de $2 \mathrm{~cm}$ e tempos de permanência de 10 ou 20 min para a casca de pinus com vermiculita. O conhecimento da dinâmica da ascensão capilar da água na subirrigação permite a otimização da altura de lâmina e do tempo para a realização de irrigações, promovendo aplicação de água com eficiência para suprir a evapotranspiração das culturas.

\section{AGRADECIMENTOS}

À Rosa Helena Aguiar, Maycon
BARRETO, CVG; TESTEZLAF, R; SALVADOR, CA. 2012. Dinâmica do potencial matricial em substratos de pinus e coco sob ação da capilaridade. Horticultura Brasileira 30: 26-31.

BIERNBAUM, JA. 1990. Get ready for subirrigation. Greenhouse Grower 8: 130-133.

CARON, J; ELRICK, DE; BEESON, R; BOUDREAU, J. 2005. Defining critical capillary rise properties for growing media in nurseries. Soil Science Society American Journal 69: 794-806.

DUMROESE, RK; PINTO, JR; JACOBS, DF; DAVIS, AS; HORIUCHI, B. 2006. Subirrigation reduces water use, nitrogen loss, and moss growth in a container nursery. Native Plants Journal 7: 253-261.

ELLIOTT, GC. 1992. A pulsed subirrigation system for small plots. HortScience 27: 71-72.

EMBRAPA - Embrapa Pecuária Sudeste. 2005. Manual de Laboratórios: Solo, Água, Nutrição animal e Alimentos. São Carlos: IICT. 313p.

FERRAREZI, RS; RIBEIRO, MD; VAN IERSEL, MW; TESTEZLAF, R. 2013. Subirrigation controlled by capacitance sensors for citrus rootstock production. HortScience 48: S142 (Abstract).

FERRAREZI, RS; SANTOS, LNS; SOUSA, ACM; PEREIRA, FFS; ELAIUY, MLC; TORREL, U; MATSURA, EE. 2012. Altura da lâmina, tempo e volume de enchimento de um equipamento de irrigação por pavio e determinação da uniformidade de distribuição de água em substratos. Bragantia 71:273-281.

FERRAREZI, RS; TESTEZLAF, R. 2016. Performance of wick irrigation system using self-compensating benches with substrates for lettuce production. Journal of Plant Nutrition 39: $1-15$.

FERRAREZI, RS; VAN IERSEL, MW; TESTEZLAF, R. 2014. Subirrigation automated by capacitance sensors for salvia production. Horticultura Brasileira 32: 314-320.

FERRAREZI, RS; VAN IERSEL, MW; TESTEZLAF, R. 2015a. Monitoring and controlling ebb-and-flow subirrigation with soil moisture sensors. HortScience 50: 447453.

FERRAREZI, RS; VAN IERSEL, MW; TESTEZLAF, R. 2015b. Uso da subirrigação para imposição de estresse hídrico em sistema semi-contínuo para medição de $\mathrm{CO}_{2}$. Ornamental Horticulture 21: 235-242.

FERRAREZI, RS; WEAVER, GM; VAN IERSEL, MW; TESTEZLAF, R. 2015c. Subirrigation: Historical overview, challenges, and future prospects. HortTechnology 25: 262-276.

GENT, MPN; MCAVOY, RJ. 2011. Water and nutrient uptake and use efficiency with partial saturation ebb and flow watering. HortScience 46: 791-798.

KIM, J; VAN IERSEL, MW; BURNETT, SE. 2011. Estimating daily water use of two petunia cultivars based on plant and environmental factor. HortScience 46: 1287-1293.

LAVIOLA, BG; MARTINEZ, HEP; MAURI, AL. 2007. Influência do nível de adubação de plantas matrizes na formação de mudas de cafeeiros em sistema hidropônico. Ciência e 
Agrotecnologia 31: 1043-1047.

LOPES, JL; GUERRINI, IA; SAAD, JC. 2007. Qualidade de mudas de eucalipto produzidas sob diferentes lâminas de irrigação e dois tipos de substrato. Revista Árvore 31: 835-843.

MARTINETTI, L; FERRANTE, A; QUATTRINI, E. 2008. Effect of drip or subirrigation on growth and yield of Solanum melongena $\mathrm{L}$. in closed systems with salty water. Research Journal of Biological Sciences 3: 467-474.

MILNER, L. 2001. Water and fertilizers management in substrates. In: IX ${ }^{\text {th }}$ INTERNATIONAL CONGRESS OF CITRUS NURSERYMEN. Anais... Riverside: International Society of Citriculture, p. 108111.

PIRE, R; PEREIRA, A. 2003. Propiedades físicas de componentes de sustratos de uso común en la horticultura del estado Lara, Venezuela: Propuesta metodológica. Bioagro 15: 55-64.

RIBEIRO, MD; FERRAREZI, RS; TESTEZLAF, R. 2014. Assessment of subirrigation performance in eucalyptus seedling production. HortTechnology 24: 231-237.

ROEBER, RU. 2010. Environmentally sound plant production by means of soilless cultivation. Comunicata Scientiae 1: 1-8.

ROUPHAEL, Y; CARDARELLI, M; REA E; BATTISTELLI, AC. 2006. Comparison of the subirrigation and drip-irrigation systems for greenhouse zucchini squash production using saline and non-saline nutrient solutions. Agricultural Water Management 82: 99-117.

SCHMAL, JL; DUMROESE, RK; DAVIS, AS; PINTO, JR; JACOBS, DF. 2011. Subirrigation for production of native plants in nurseries - Concepts, current knowledge, and implementation. Native Plants Journal 12: 81-93.

THEBALDI, MS. 2011. Irrigação de mudas de espécies florestais nativas produzidas em tubetes. Lavras: UFLA. 93p (Dissertação mestrado).

UVA, WFL; WEILER, TC; MILLIGAN, RA.
1998. A survey on the planning and adoption of zero runoff subirrigation systems in greenhouse operations. HortScience 36: 167-173.

VAN DER GAAG, DJ; KERSSIES, A; LANSER, C. 2001. Spread of phytophthora root and crown rot in saintpaulia, gerbera and spathiphyllum pot plants in ebb-and-flowsystems. European Journal of Plant Pathology 107: 535-542.

VAN IERSEL, MW; KANG, JG. 2002. Nutrient solution concentration affects whole-plant $\mathrm{CO}_{2}$ exchange and growth of subirrigated pansy. Journal of the American Society for Horticultural Science 127: 423-429.

ZANETTI, M; FERNANDES, C; CAZETTA, JO; CORÁ, JE; MATTOS JUNIOR, D. 2003. Características físicas de substratos para a produção de mudas cítricas sob telado. Revista Laranja 24: 519-530.

ZHENG, Y; GRAHAM, TH; RICHARD, S; DIXON, M. 2004. Potted gerbera production in a subirrigation system using low-concentration nutrient solutions. HortScience 39: 1283-1286. 